\section{(6) OPEN ACCESS}

\title{
Hemorrhagic complications after prasugrel (Effient) therapy for vascular neurointerventional procedures
}

\author{
S Hassan Akbari, ${ }^{1}$ Matthew R Reynolds, ${ }^{1}$ Yasha Kadkhodayan, ${ }^{2}$ \\ DeWitte T Cross III, ${ }^{1,2}$ Christopher J Moran ${ }^{1,2}$
}

${ }^{1}$ Department of Neurological Surgery, Washington University School of Medicine, St Louis, Missouri, USA

2Department of Radiology, Mallinckrodt Institute of Radiology, Washington University School of Medicine, St Louis, Missouri, USA

\section{Correspondence to}

Dr M R Reynolds,

Barnes-Jewish Hospital,

Department of Neurological

Surgery, Campus Box 8057, 660

South Euclid Avenue, St. Louis,

MO 63310, USA:

reynoldsm@wudosis.wustl.edu

SHA and MRR contributed equally to this work.

Received 28 February 2012 Revised 2 April 2012 Accepted 9 April 2012 Published Online First 3 May 2012

\section{SLinked}

- http://dx.doi.org/10.1136/ neurintsurg-2012-010302

- http://dx.doi.org/10.1136/ neurintsurg-2012-010406

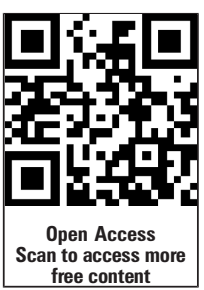

\begin{abstract}
Introduction Dual antiplatelet therapy (DAPT) with aspirin and a thienopyridine (eg, clopidogrel) prevents stent related thromboembolic events in cardiac patients and is frequently utilized during neurointerventional surgery. However, recent data suggest that many patients exhibit clopidogrel resistance. Prasugrel-a newer thienopyridine-lowers the rate of cardiac stent thromboses in clopidogrel non-responders but a paucity of data exist regarding its safety and efficacy in neurointerventional surgery.

Methods All patients undergoing neurointerventional surgery by a single interventionalist (CJM) over a 20 month period were retrospectively identified. Charts were reviewed for pre- and post-procedural DAPT regimens, pre-procedural coagulation parameters and procedural complications.
\end{abstract}

Results 76 patients received pre- and post-procedural DAPT for endovascular treatment of an intracerebral aneurysm, dural arteriovenous fistula or intra/extracranial arterial stenosis. 51 patients underwent 55 total procedures and were treated with aspirin/clopidogrel; 25 patients underwent 31 total procedures and were treated with aspirin/prasugrel. Those patients who received aspirin/prasugrel DAPT were identified preprocedurally to be clopidogrel non-responders. Both treatment groups had a similar percentage of patients undergoing aneurysm coiling, stent assisted aneurysm coiling, aneurysm Onyx embolization, aneurysm pipeline embolization device treatment, extra/intracranial carotid artery angioplasty and stenting, and dural arteriovenous fistula coil embolization. A total of eight (9.3\%) hemorrhagic complications were observed, two $(3.6 \%)$ in the aspirin/clopidogrel group and six (19.4\%) in the aspirin/prasugrel group $(p=0.02)$. No differences were noted in hemorrhage rates for each procedure between treatment groups, nor were there any differences in thrombotic complications between groups.

Conclusion Our results suggest that DAPT with aspirin/ prasugrel may predispose to a higher risk of hemorrhage during neurointerventional surgery compared with DAPT with aspirin/clopidogrel.

\section{INTRODUCTION}

Endovascular therapy has evolved as an essential tool for the treatment of complex neurovascular diseases. Despite significant advances in this area, however, the risk of thromboembolic complications has been estimated at $8 \%$ due to the thrombogenic nature of foreign guidewires and endovascular implants. ${ }^{1}$ Literature from the field of interven- tional cardiology suggests that adding aspirin and clopidogrel dual antiplatelet therapy (DAPT) to post-procedural management minimizes the risk of thromboembolic complications ${ }^{2}$ and is more beneficial than single agent therapy with aspirin alone. $^{3-5}$ As a result, DAPT with full dose aspirin (325 mg orally daily) and clopidogrel (75 mg orally daily) has also been recommended for neurointerventional surgery. ${ }^{6} 7$

It has been estimated that approximately $30 \%$ of patients exhibit clopidogrel resistance. ${ }^{8} 9$ Importantly, cardiology studies suggest that clopidogrel non-responders exhibit a significantly higher rate of stent thrombosis than those patients who respond to this therapy $(8.6 \%$ vs $2.3 \%$, respectively; $\mathrm{p}<0.001) .{ }^{10}$ In a prospective study of over 800 patients, pre-procedural platelet aggregation was associated with a 6.7-fold risk of 30 day adverse events, including myocardial infarction, target lesion revascularization and death $(p<0.03) .{ }^{11}$ Those patients unresponsive to clopidogrel may be treated with newer generation antiplatelet agents. Prasugrel (Effient) is a third generation oral thienopyridine that irreversibly inhibits the $\mathrm{P}_{2} \mathrm{Y}_{12} \mathrm{ADP}$ receptor on the surface of platelets and decreases platelet aggregation. ${ }^{12}$ Prasugrel is a prodrug that is rapidly metabolized to a pharmacologically active metabolite with a plasma half-life of $\sim 4$ h. ${ }^{13}$ While the medication costs for clopidogrel and prasugrel are similar, ${ }^{14}$ prasugrel affords more potent and rapid inhibition of platelet aggregation ${ }^{15}$ and decreased intersubject response variability. ${ }^{16}$

In randomized studies, DAPT with aspirin/ prasugrel was associated with a $30 \%$ increase in the relative risk of bleeding (including fatal bleeding) compared with aspirin/clopidogrel, without a significant difference in mortality. ${ }^{17} 18$ In another prospective multicentre trial of 396 patients, Armero et al ${ }^{19}$ observed a bleeding rate of $13.6 \%$ $(3.7 \%$ with major life-threatening bleeding) in patients treated with aspirin/prasugrel DAPT for acute coronary syndrome. To date, however, there have been no studies examining the safety and efficacy of prasugrel for neurointerventional procedures. In this report, we detail our experience using DAPT with aspirin/prasugrel in this patient population.

\section{METHODS}

Following approval by the Washington University Institutional Review Board, we retrospectively identified 115 consecutive subjects who underwent an interventional neuroradiology procedure at 
Washington University School of Medicine (Mallinckrodt Institute of Radiology, St Louis, Missouri, USA) by a single interventionalist (CJM) between 15 February 2010 and 31 October 2011. Subjects treated for intracranial aneurysms, arteriovenous malformations, dural arteriovenous fistula or intra/extracranial stenosis and who received DAPT during the pre- and postprocedural periods were included.

Patient charts were retrospectively reviewed for pre- and postprocedure antiplatelet and anticoagulation therapy, as well as preoperative platelet counts, platelet function studies and coagulation parameters. Peri-procedural activated clotting times were also analyzed. All patients were loaded with aspirin (325 mg orally daily) and clopidogrel (Plavix, Bristol-Myers Squibb/Sanofi Pharmaceuticals, Bridgewater, NJ, USA) (75 mg orally daily) at least 7 days prior to their procedures. Those patients who exhibited less than $40 \%$ platelet inhibition $\left(\mathrm{P}_{2} \mathrm{Y}_{12}\right.$ $\mathrm{ADP}$ receptor inhibition) on clopidogrel, as determined by platelet screening analysis (VerifyNow, Accumetrics, San Diego, California, USA), were loaded with prasugrel (Effient; Eli Lilly, Indianapolis, IN, USA/Daiichi Sankyo) just prior to the procedure (60 mg orally once). These patients stopped their clopidogrel and were continued on prasugrel $10 \mathrm{mg}$ orally daily after the procedure. Patients who were responsive to clopidogrel (greater than $40 \%$ platelet inhibition) were continued on clopidogrel (75 mg orally daily) in addition to full dose aspirin (325 mg orally daily). In some cases, a lower dose of aspirin (81 mg orally daily) was given. VerifyNow platelet inhibition assays were not routinely performed in those patients exhibiting clopidogrel resistance after prasugrel treatment. Furthermore, genetic testing for cytochrome P450 polymorphisms was not performed in patients demonstrating laboratory evidence of clopidogrel resistance. All patients were heparinized during their procedures. Those undergoing aneurysm treatment and intra/ extracranial stenosis treatment were therapeutically anticoagulated with an activated clotting time of at least twice baseline.

Patient demographics, procedure type, and pre- and postprocedural antiplatelet regimens were recorded. Charts were analyzed for all procedural neurointerventional complications. Hemorrhagic complications were defined according to the previously published Thrombolysis in Myocardial Infarction criteria. ${ }^{20}$ Specifically, major bleeding was defined as intracerebral hemorrhage or clinically overt bleeding (including imaging) associated with a decrease in hemoglobin of $\geq 5 \mathrm{~g} / \mathrm{dl}$; minor bleeding was defined as any clinically overt bleeding (including imaging) associated with a decrease in hemoglobin of $3-5 \mathrm{~g} / \mathrm{dl}$. Groin hematomas without hemodynamic alterations not requiring a blood transfusion and mild, self-limiting episodes of epistaxis were not included as hemorrhagic complications.

Statistical analyses were performed using a Student's two tailed $t$ test or Wilcoxon rank sum test as appropriate for continuous variables and a Fisher's exact test for categorical variables. A p value $\leq 0.05$ was considered significant.

\section{RESULTS}

Seventy-six patients were identified who received DAPT with either aspirin/clopidogrel $(n=51)$ or aspirin/prasugrel $(n=25)$ during and after their neurointerventional procedures. In both treatment groups, some patients underwent multiple procedures resulting in a total of 86 interventions $(n=55$ for the aspirin/ clopidogrel group; $n=31$ for the aspirin/prasugrel group) (table 1). The average patient age at the time of intervention was $61.2 \pm 12.4$ years for the aspirin/clopidogrel group and $59.4 \pm 14.8$ years for the aspirin/clopidogrel group $(p=0.565)$. In the aspirin/clopidogrel DAPT group, 17 (30.9\%) patients underwent aneurysm coiling, 22 (40\%) patients underwent stent assisted aneurysm coiling, three (5.5\%) patients underwent aneurysm Onyx embolization, seven (12.7\%) patients underwent aneurysm pipeline embolization device (PED) placement, one patient (1.8\%) underwent dural arteriovenous fistula coil embolization and five patients (9.1\%) underwent extra- or intracranial carotid angioplasty and stenting (table 1). For the aspirin/prasugrel DAPT group, five (16.1\%) patients had aneurysm coil embolization, eight $(25.8 \%)$ patients had stent assisted aneurysm coiling, three $(9.7 \%)$ patients had aneurysm Onyx embolization, nine (29\%) patients had aneurysm PED treatment and six (19.4\%) patients had extra- or intracranial carotid angioplasty and stenting. There were no significant differences in the number of patients undergoing each procedure between treatment groups.

A total of eight (9.3\%) hemorrhagic complications were observed. Hemorrhages were seen in two $(3.6 \%)$ patients treated with aspirin/clopidogrel DAPT and in six (19.4\%) patients treated with aspirin/prasugrel DAPT $(p=0.02)$ (table 2). Excluding one patient in the aspirin/prasugrel DAPT group who experienced a basilar artery perforation, the difference in hemorrhage rates between the DAPT groups trended towards significance but did not reach statistical significance $(p=0.09)$. In the aspirin/clopidogrel group, hemorrhage was observed in one $(33.3 \%)$ patient treated with Onyx aneurysm embolization and one $(14.3 \%)$ patient treated with aneurysm PED placement. For the aspirin/prasugrel group, hemorrhagic events were seen in one $(22.6 \%)$ patient treated with aneurysm coil embolization, one $(12.5 \%)$ patient treated with aneurysm stent assisted coil embolization, two (22.2\%) patients treated with aneurysm PED placement, one $(33.3 \%)$ patient treated with extracranial carotid angioplasty and stenting, and one (50\%) patient treated with intracranial carotid angioplasty and stenting (table 2). No statistically significant differences in hemorrhage rates were observed between treatment groups for each procedure. There were no significant differences in the rate of neurological (eg, intracerebral) and non-neurological (eg, extracerebral) hemorrhage between treatment groups ( $p<0.13$ in both cases).

In the aspirin/clopidogrel group, one patient experienced a groin hematoma with retroperitoneal extension requiring a blood transfusion after Onyx embolization of a right

Table 1 Patient demographics and procedures performed

\begin{tabular}{|c|c|c|c|c|c|c|c|c|c|c|}
\hline Treatment group & n & $\begin{array}{l}\text { Gender } \\
\text { (n (\%)) }\end{array}$ & $\begin{array}{l}\text { Age } \\
\text { (years) }\end{array}$ & $\begin{array}{l}\text { Anx } \\
\text { coil (n (\%)) }\end{array}$ & $\begin{array}{l}\text { Anx stent } \\
\text { coil }(\mathbf{n}(\%))\end{array}$ & $\begin{array}{l}\text { Anx Onyx } \\
\text { embo (n (\%)) }\end{array}$ & $\begin{array}{l}\text { Anx PED } \\
\text { (n (\%)) }\end{array}$ & $\begin{array}{l}\text { Extracranial carotid } \\
\text { angioplasty and } \\
\text { stenting (n }(\%))\end{array}$ & $\begin{array}{l}\text { Intracranial carotid } \\
\text { angioplasty and } \\
\text { stenting (n (\%)) }\end{array}$ & $\begin{array}{l}\text { dAVF coil } \\
\text { embo }(n(\%))\end{array}$ \\
\hline Aspirin and clopidogrel & 55 & $11 \mathrm{M}(20)$ & $61.2 \pm 12.4$ & $17(30.9)$ & $22(40.0)$ & $3(5.5)$ & $7(12.7)$ & $3(5.5)$ & $2(3.6)$ & $1(1.8)$ \\
\hline \multirow[t]{2}{*}{ Aspirin and prasugrel } & 31 & $7 \mathrm{M}(23)$ & $59.4 \pm 14.8$ & $5(16.1)$ & $8(25.8)$ & $3(9.7)$ & $9(29.0)$ & $6(19.4)$ & $0(0)$ & $0(0)$ \\
\hline & & 24 F (77) & & & & & & & & \\
\hline $\mathrm{p}$ Value & & & 0.565 & 0.198 & 0.241 & 0.663 & 0.084 & 0.065 & - & - \\
\hline
\end{tabular}

Anx, aneurysm; dAVF, dural arteriovenous fistula; embo, embolization; PED, pipeline embolization device. 
Table 2 Hemorrhagic complications between treatment groups

\begin{tabular}{|c|c|c|c|c|c|c|c|c|}
\hline Treatment group & $\begin{array}{l}\text { Total } \\
\text { (n (\%)) }\end{array}$ & $\begin{array}{l}\text { Anx coil } \\
\text { (n }(\%))\end{array}$ & $\begin{array}{l}\text { Anx stent } \\
\text { coil }(\mathbf{n}(\%))\end{array}$ & $\begin{array}{l}\text { Anx Onyx } \\
\text { embo (n (\%)) }\end{array}$ & $\begin{array}{l}\text { Anx PED } \\
\text { (n }(\%))\end{array}$ & $\begin{array}{l}\text { Extracranial carotid } \\
\text { angioplasty and } \\
\text { stenting }(\mathrm{n}(\%))\end{array}$ & $\begin{array}{l}\text { Intracranial carotid } \\
\text { angioplasty and } \\
\text { stenting (n (\%)) }\end{array}$ & $\begin{array}{l}\text { dAVF coil } \\
\text { embo (n }(\%))\end{array}$ \\
\hline Aspirin and clopidogrel & $2(3.6)$ & $0(0)$ & $0(0)$ & $1(33.3)$ & $1(14.3)$ & $0(0)$ & $0(0)$ & $0(0)$ \\
\hline $\mathrm{p}$ Value & 0.02 & 0.24 & 0.27 & - & 1.0 & - & - & - \\
\hline
\end{tabular}

Anx, aneurysm; dAVF, dural arteriovenous fistula; embo, embolization; PED, pipeline embolization device.

paraclinoid aneurysm. Another patient, treated with PED for a left cavernous carotid aneurysm, presented 5 days after treatment with headache and was found to have an anterior interhemispheric subarachnoid hemorrhage. In the aspirin/prasugrel group, one patient had a basilar artery perforation with subarachnoid hemorrhage, intraventricular hemorrhage and symptomatic hydrocephalus; one patient had a right cervical carotid perforation with neck hematoma as well as spontaneous flank, groin and scrotal hematomas; one patient had a left frontal intraparenchymal hemorrhage and upper gastrointestinal bleed; one patient experienced a right cerebellar intraparenchymal bleed; and two patients had brisk epistaxis requiring surgical packing and blood transfusion (table 3 ).

A single thromboembolic complication (transient ischemic attack) was observed in the aspirin/prasugrel DAPT treatment group following stent assisted coiling of a left superior hypophyseal aneurysm. There were no significant differences in the rate of thromboembolic events between the two DAPT treatment groups. All patients had normal platelet counts (>100000 K/cumm) and coagulation parameters (international normalized ratio $<1.3 \mathrm{~s}$, partial thromboplastin time $<40 \mathrm{~s}$ ) prior to their procedures.

\section{PRASUGREL RELATED HEMORRHAGIC COMPLICATIONS: CASE SUMMARIES \\ Case No 1}

A woman in her fourth decade of life with a history of moya moya disease status post encephaloduroarteriosynangiosis in 1997 presented with headache for 2 weeks and was found to have an unruptured $7 \times 4 \mathrm{~mm}$ basilar apex aneurysm (figure $1 \mathrm{~A}$ ). She was started on full dose aspirin and clopidogrel therapy and underwent stent assisted endovascular coiling of the aneurysm. She was loaded with prasugrel (60 mg orally) on the day of the endovascular treatment due to clopidogrel resistance. During stent deployment, the microwire was noted to pass through the lateral wall of the basilar artery with contrast extravasation (figure 1B). Serial injections demonstrated decreased contrast extravasation over time. The patient's left pupil became fixed and dilated. A non-contrast head CT showed extensive subarachnoid hemorrhage with intraventricular hemorrhage and obstructive hydrocephalus (figure $1 \mathrm{D}-\mathrm{F}$ ). She received intravenous mannitol with resolution of her pupillary abnormality. Neurosurgery was consulted and a ventriculostomy was placed. On post-procedure day (PPD) 1, the patient was continued on full dose aspirin and prasugrel. She developed a left sixth nerve palsy that gradually resolved. Her ventriculostomy was weaned and discontinued. She experienced a meaningful neurological recovery and was discharged on PPD 15. She presented several months later with headaches and was noted to have hydrocephalus on follow-up imaging. A ventriculoperitoneal shunt was placed with resolution of her symptoms. She later presented electively for definitive coil embolization of her aneurysm (figure 1C).

\section{Case No 2}

A man in his sixth decade of life who presented with decreased vision in his right eye was found to have a giant right cavernous carotid aneurysm (figure 2A). The patient was placed on full dose aspirin and clopidogrel prior to endovascular therapy. $\mathrm{He}$ was loaded with prasugrel ( $60 \mathrm{mg}$ orally) on the day of treatment due to clopidogrel resistance. He presented for elective placement of PEDs to the right cavernous segment. The procedure was complicated by perforation of the proximal right cervical carotid artery with active contrast extravasation (figure 2B) and development of a right neck hematoma. Several PEDs were quickly deployed across the aneurysm neck (figure 2C); an additional PED was placed across the perforated segment to control the hemorrhage. A non-contrast neck CT showed soft tissue stranding and likely hemorrhage adjacent to

Table 3 Hemorrhagic complications within aspirin/prasugrel treatment group

\begin{tabular}{|c|c|c|c|c|c|c|}
\hline $\begin{array}{l}\text { Patient } \\
\text { No }\end{array}$ & $\begin{array}{l}\text { Decade } \\
\text { of life }\end{array}$ & Gender & Procedure & $\begin{array}{l}\text { Pre-procedural antiplatelet } \\
\text { therapy }\end{array}$ & $\begin{array}{l}\text { Post-procedural antiplatelet } \\
\text { therapy }\end{array}$ & Complications \\
\hline 1 & 4th & $\mathrm{F}$ & $\begin{array}{l}\text { Stent assisted coiling } \\
\text { of basilar apex Anx }\end{array}$ & $\begin{array}{l}\text { ASA } 325 \text { mg PO daily; prasugel } \\
60 \text { mg PO load }\end{array}$ & $\begin{array}{l}\text { ASA } 325 \text { mg PO daily; prasugrel } \\
10 \text { mg PO daily }\end{array}$ & $\begin{array}{l}\text { Basilar artery perforation, SAH, } \\
\text { IVH, hydrocephalus }\end{array}$ \\
\hline 2 & 6 th & $\mathrm{M}$ & $\begin{array}{l}\text { PED treatment of right } \\
\text { ICA Anx }\end{array}$ & $\begin{array}{l}\text { ASA } 325 \text { mg PO daily; prasugrel } \\
60 \text { mg PO load }\end{array}$ & $\begin{array}{l}\text { ASA } 325 \text { mg PO daily; prasugrel } \\
10 \text { mg PO daily }\end{array}$ & $\begin{array}{l}\text { Right cervical ICA perforation with } \\
\text { neck hematoma; spontaneous flank, } \\
\text { groin and scrotal hematomas }\end{array}$ \\
\hline 3 & 8th & $\mathrm{F}$ & $\begin{array}{l}\text { PED treatment of left } \\
\text { cavernous carotid artery Anx }\end{array}$ & $\begin{array}{l}\text { ASA } 325 \text { mg PO daily; prasugrel } \\
60 \text { mg PO load }\end{array}$ & Prasugrel 10 mg P0 daily & $\begin{array}{l}\text { Left frontal IPH; upper GI bleed } \\
\text { requiring blood transfusion }\end{array}$ \\
\hline 4 & 6th & $\mathrm{F}$ & $\begin{array}{l}\text { Coiling of left superior } \\
\text { cerebellar artery Anx }\end{array}$ & $\begin{array}{l}\text { ASA } 325 \text { mg PO daily; prasugrel } \\
60 \text { mg PO load }\end{array}$ & $\begin{array}{l}\text { ASA } 81 \text { mg PO daily; clopidogrel } \\
75 \text { mg PO daily; prasugrel } 10 \mathrm{mg} \\
\text { PO PPD 1-2 }\end{array}$ & Right cerebellar IPH \\
\hline 5 & 5th & M & $\begin{array}{l}\text { Balloon angioplasty and } \\
\text { stenting of right ICA stenosis }\end{array}$ & $\begin{array}{l}\text { ASA } 325 \text { mg PO daily; prasugrel } \\
60 \text { mg PO load }\end{array}$ & $\begin{array}{l}\text { ASA } 325 \text { mg PO daily; prasugrel } \\
10 \text { mg PO daily }\end{array}$ & Epistaxis requiring nasal packing \\
\hline 6 & 6th & $\mathrm{M}$ & $\begin{array}{l}\text { Balloon angioplasty and } \\
\text { stenting of right ICA stenosis }\end{array}$ & $\begin{array}{l}\text { ASA } 325 \text { mg PO daily; prasugrel } \\
60 \text { mg PO load }\end{array}$ & $\begin{array}{l}\text { ASA } 325 \text { mg PO daily; prasugrel } \\
10 \text { mg PO daily }\end{array}$ & $\begin{array}{l}\text { Epistaxis requiring nasal packing } \\
\text { and blood transfusion }\end{array}$ \\
\hline
\end{tabular}

Anx, aneurysm; ASA, acetylsalicylic acid (aspirin); ICA, internal carotid artery; IPH, intraparenchymal hemorrhage; IVH, intraventricular hemorrhage; PED, pipeline embolization device; PO, orally; PPD, post-procedure day; SAH, subarachnoid hemorrhage. 
Figure 1 (A) Anteroposterior view of the cerebral vasculature following a left vertebral artery contrast injection. A large aneurysm at the basilar apex is visualized. (B) Active extravasation of contrast agent from the lateral wall of the basilar artery (denoted by arrowhead). (C) Repeat cerebral catheter angiogram with injection of the left vertebral artery showing near complete aneurysm occlusion after endovascular coil embolization. (D-F) Serial sections from a non-contrast head CT showing diffuse hyperdensity within the basilar cisterns, bilateral Sylvian fissures and intraventricular space with obstructive hydrocephalus.
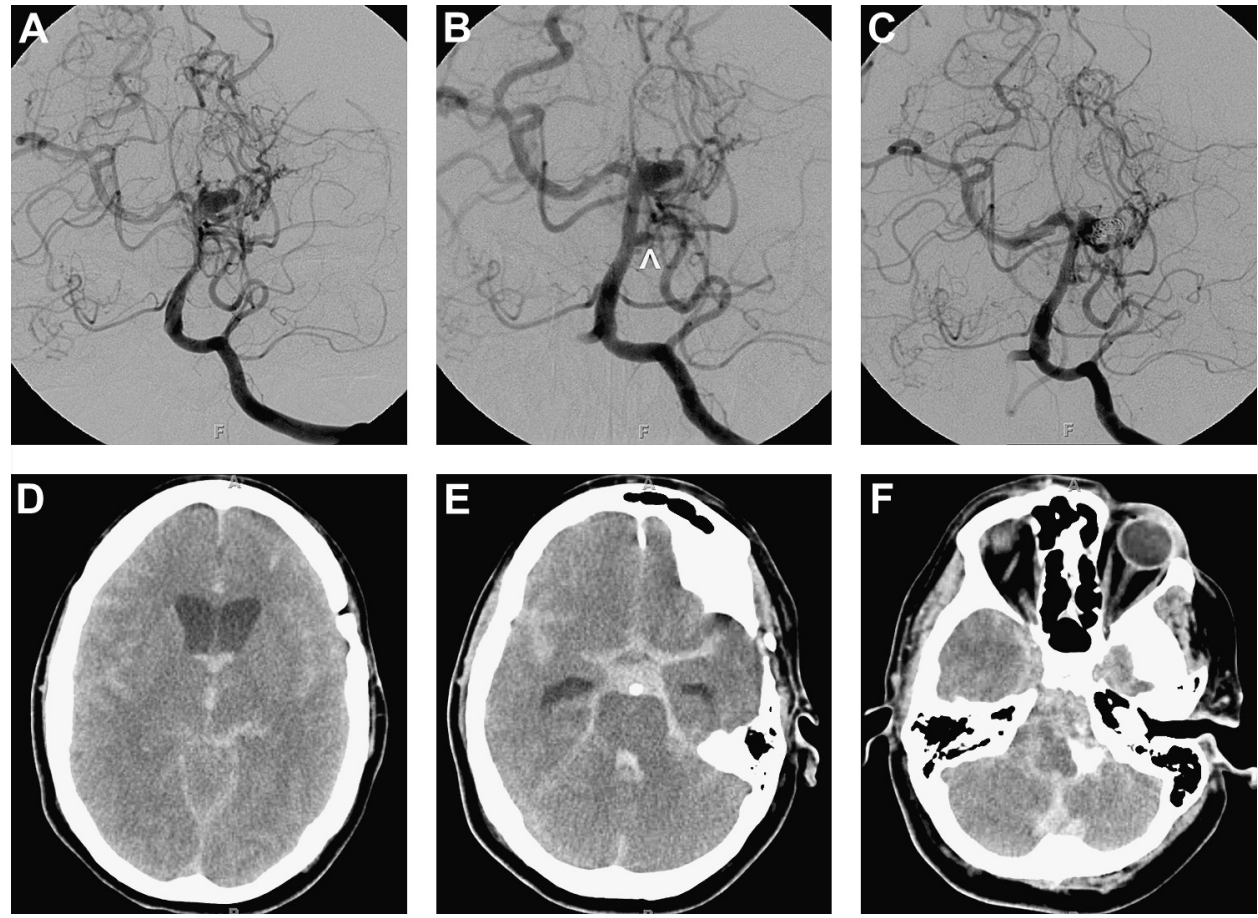

the internal carotid artery (figure 2D). The patient remained intubated and was transferred to the intensive care unit. On PPD 2, he became hemodynamically unstable and was found to have a large rectus sheath and right groin hematoma (figure 2E). He was taken to the operating room with vascular surgery for placement of a covered iliofemoral stent for hemorrhage control. On PPD 3, he was restarted on full dose aspirin and prasugrel. The same day, the patient was noted to be less responsive, tachypneic and tachycardic. Chest CT demonstrated large bilateral pulmonary emboli. He was started on a bivalirudin intravenous drip. He was extubated on PPD 5. Coumadin therapy was started for deep venous thromboses and pulmonary emboli. He was discharged to a rehabilitation facility on PPD 15 and had a meaningful neurological recovery.

\section{Case No 3}

A woman in her eighth decade of life with a history of a left ophthalmic artery aneurysm status post coiling 25 years previously presented with left-sided ophthalmoplegia and ptosis secondary to mass effect from a recurrent, giant left internal carotid artery aneurysm (figure 3A). She was started on full dose aspirin and clopidogrel prior to her procedure. She was loaded with prasugrel (60 mg orally) on the day of the endovascular treatment secondary to clopidogrel resistance. The patient underwent placement of multiple PEDs across the aneurysm neck without complications (figure 3B,C). Full dose aspirin and prasugrel were continued on PPD 1 . Her hospital course was uncomplicated and she was discharged to home on PPD 3. One month after her procedure she was admitted to the intensive care unit with an upper gastrointestinal bleed and severe anemia requiring transfusion with 6 units of packed red blood cells. Upper endoscopy showed gastric erosion that was treated by thermocoagulation. Her antiplatelet regimen was held. A head CT performed at this time showed an asymptomatic right frontal intraparenchymal hemorrhage (figure 3D). The patient was started on ticlopidine (250 mg orally twice daily) and later discharged in a stable condition.

\section{Case No 4}

A woman in her sixth decade of life with a 10 year history of an asymptomatic left superior cerebellar artery aneurysm presented with a 2 month history of diplopia. Catheter angiography revealed a $12 \times 22 \mathrm{~mm}$ left superior cerebellar artery aneurysm (figure 4A). The patient was loaded with clopidogrel (300 mg orally) and then started on full dose aspirin and clopidogrel. She was loaded with prasugrel (60 mg orally) on the day of the neurointerventional treatment due to clopidogrel resistance and underwent successful endovascular coiling (figure 4B). Her aspirin and prasugrel were continued post-procedurally. On PPD 2 , she developed a severe occipital headache with nausea and vomiting. Head CT showed a small intraparenchymal hemorrhage in the right cerebellar hemisphere (figure 4C). Antiplatelet therapy was held and she was transfused with single donor platelets. She was discharged in a stable condition on PPD 6 without antiplatelet agents (restarted at a later date).

\section{Case No 5}

A man in his sixth decade of life with a recent history of ischemic stroke and residual left-sided hemiparesis presented with severe (>99\%) right internal carotid artery stenosis. He was started on full dose aspirin and clopidogrel prior to endovascular treatment. Given his unresponsiveness to clopidogrel, he was loaded with prasugrel (60 mg orally) immediately before the procedure. He underwent a balloon angioplasty with stent placement without complications. While in the recovery room, he developed brisk epistaxis. Otolaryngology was consulted and his right nare was packed. He developed continued epistaxis that night requiring repacking of the nare. The packings were removed and he was restarted on full dose aspirin and prasugrel on PPD 3. Despite a decrease in hematocrit (44.9\% to $30 \%)$, he did not require a blood transfusion. He was discharged in a stable condition.

\section{Case No 6}

A man in his fifth decade of life presented with a left facial droop, left-sided hemiparesis and dysarthria. Head CT and MRI 
Figure 2 (A) Anteroposterior view of the cerebral circulation following a right common carotid artery injection demonstrating a large cavernous carotid aneurysm. (B) Active extravasation from the proximal right cervical carotid artery (denoted by arrowhead). (C) Anteroposterior skull radiographs showing the pipeline embolization device deployed within the cavernous carotid artery. (D) Non-contrast neck CT demonstrating soft tissue stranding and likely hematoma in the area adjacent to the carotid injury (asterisks denote cervical carotid artery and internal jugular vein). (E) Non-contrast pelvic CT showing a large hematoma centered within the right abdominal wall.
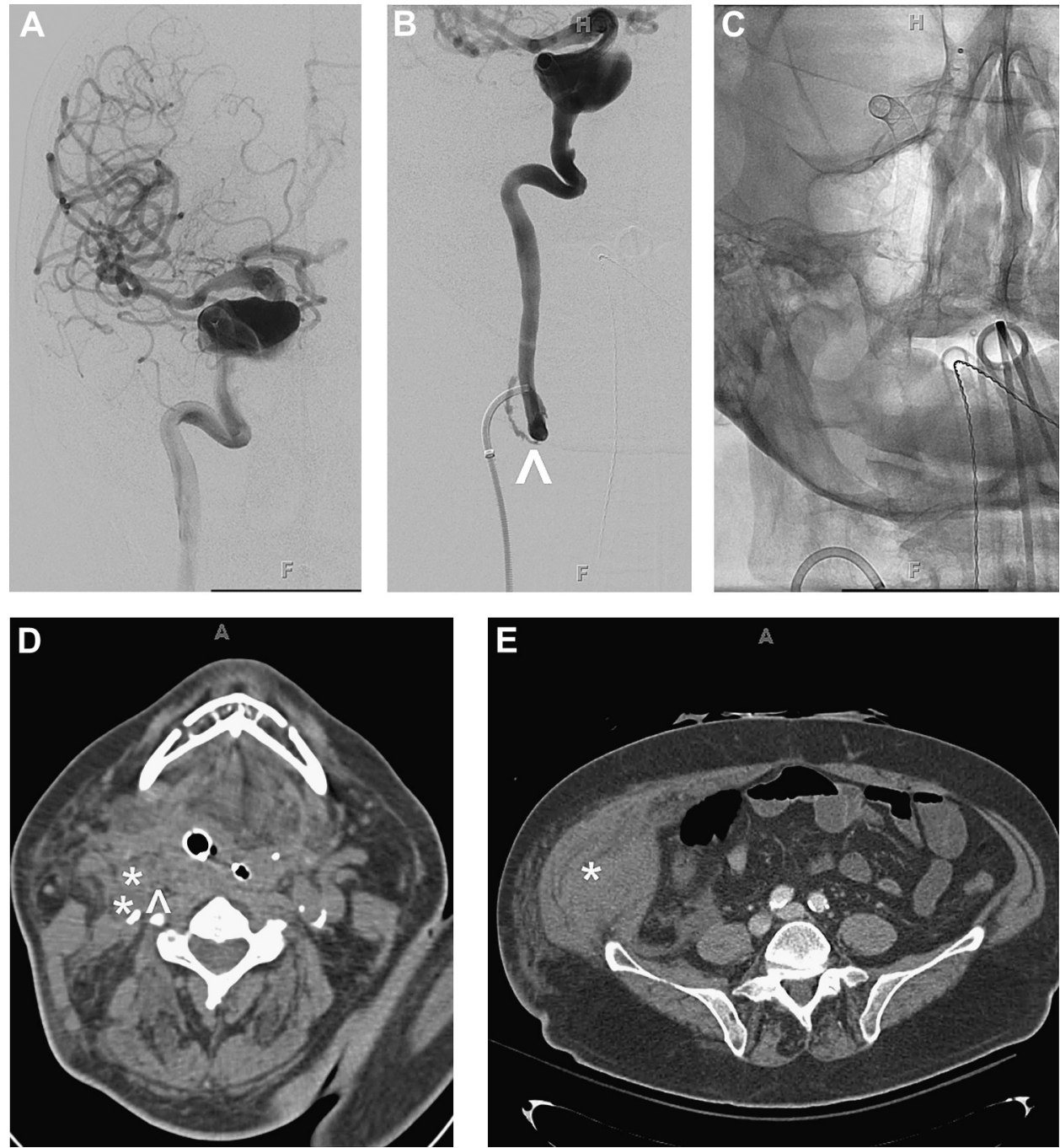

studies were suggestive of an ischemic stroke in the distribution of the right middle cerebral artery. Cerebral catheter angiography showed a near occlusion of the right internal carotid artery. Given the patient's multiple medical comorbidities, he was deemed a candidate for carotid stenting. Full dose aspirin and clopidogrel were started. He was loaded with prasugrel $(60 \mathrm{mg}$ orally) on the day of the endovascular treatment due to clopidogrel resistance. He underwent carotid artery stenting without complications. Full dose aspirin and prasugrel were continued post-procedurally. He was discharged home without incident.

On PPD 20, he experienced an episode of brisk epistaxis requiring readmission and posterior nasal packing. While hemodynamically stable, he was transfused 2 units of packed red blood cells for a hematocrit $<23 \%$. He was later discharged in a stable condition.

\section{DISCUSSION}

In the present study, we observed an increased rate of hemorrhage in patients treated with aspirin/prasugrel compared with those treated with aspirin/clopidogrel $(19.4 \%$ vs $3.6 \%$,
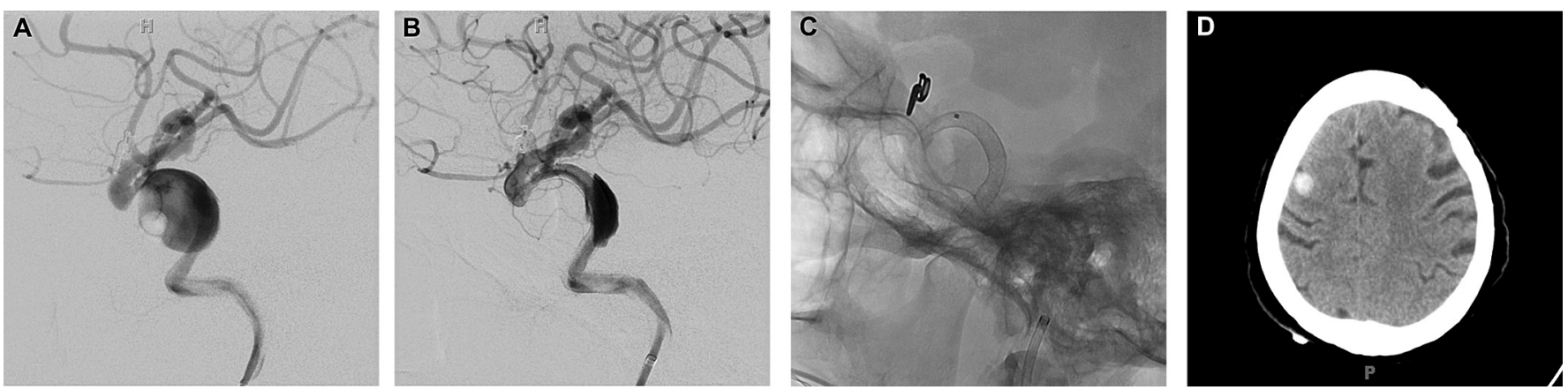

Figure 3 (A) Anteroposterior view of the cerebral circulation following a left common carotid artery injection showing a large aneurysm arising from the cavernous carotid artery. (B) Repeat cerebral catheter angiography immediately following deployment of several pipeline embolization devices (PEDs) showing reduction of contrast within the aneurysm. (C) Lateral skull radiographs showing the PED deployed within the cavernous carotid artery. (D) Non-contrast head CT showing a small right frontal intraparenchymal hemorrhage. 
Figure 4 (A) Anteroposterior view of the cerebral circulation following a right vertebral artery contrast injection showing a large left superior cerebellar artery aneurysm. (B) Repeat cerebral catheter angiogram following near complete endovascular coil embolization. (C) Non-contrast head CT demonstrating a small focus of intraparenchymal hemorrhage within the right cerebellar hemisphere.
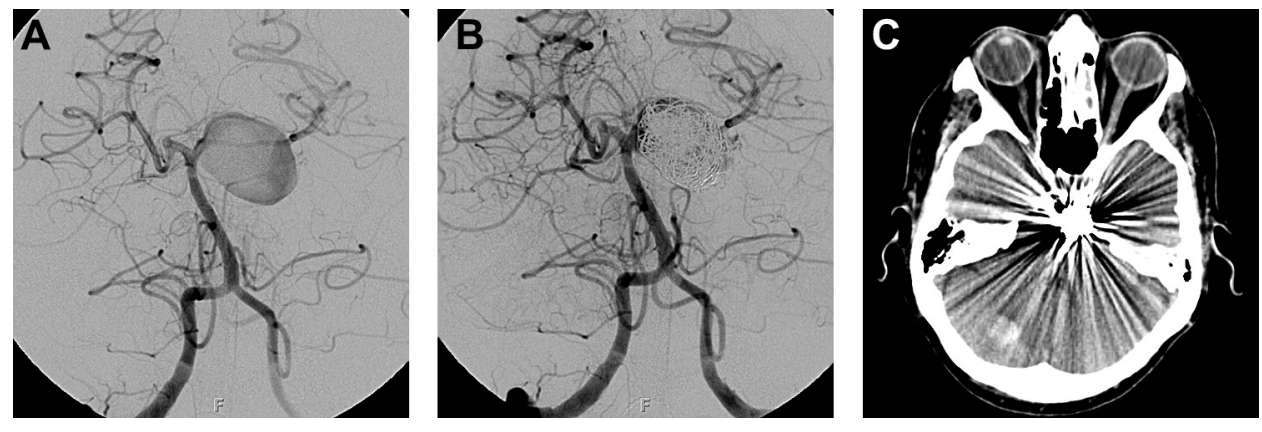

respectively). There were no differences in hemorrhage rate for each procedure between the DAPT treatment groups, and there were no differences in the rate of thromboembolic complications between groups. The incidence of hemorrhage seen in our patients treated with aspirin/prasugrel was higher than that reported in the interventional cardiology literature $(\sim 2-4 \%)^{18} 19$ but this may be attributable to differences in vessel tortuosity, hemodynamics and vessel fragility between the two vascular networks.

Notably, if we excluded one aspirin/prasugrel DAPT patient who experienced a basilar artery perforation during aneurysm coiling-given the possibility that the hemorrhage was caused by a technical complication rather than from excessive platelet inhibition-our data trended towards statistical significance but did not reach significance. We elected to include this patient in our study for several reasons. First, nearly all other variables between DAPT treatment groups were similar (eg, patient characteristics, case length, procedure type, technical complexity, personnel involved, etc). This observation suggests that the antiplatelet regimen-and not the technical aspects of the procedure-may be responsible for the increased hemorrhage rate in the aspirin/prasugrel group. Second, there can be varying degrees of vascular injury during endovascular procedures ranging from minor clinically insignificant arterial wall injury to frank perforation with active extravasation. Therefore, it is possible that the magnitude of platelet inhibition achieved with aspirin/prasugrel could convert a clinically occult vascular injury into a major hemorrhagic event.

Data from subgroup analyses comparing the efficacy of aspirin/clopidogrel with aspirin/prasugrel DAPT in patients with acute coronary syndrome demonstrate that age (>75 years), prior thromboembolic events, bleeding propensity, body weight $(<60 \mathrm{~kg})$ and various concomitant medication usage may increase the risk of hemorrhage from thienopyridine therapy. ${ }^{21}$ For this reason, it has been recommended by some authorities that patients at increased risk of bleeding from prasugrel be treated with a lower maintenance dose $(5 \mathrm{mg}$ orally daily). ${ }^{21}$ Notably, the majority of patients who experienced major hemorrhagic complications from prasugrel therapy in our series had few, if any, of these risk factors. Further, given the lack of prospective data regarding the safety and efficacy of prasugrel at doses other than those utilized in this series (eg, $60 \mathrm{mg}$ orally loading dose, with $10 \mathrm{mg}$ orally daily maintenance dose), we adhered to this regimen-with some exceptions-even in the setting of bleeding complications (see table 3 and case summaries).

To our knowledge, there has been only one prior case report examining the use of aspirin/prasugrel DAPT in the setting of neurointerventional surgery. Leslie-Mazwi et al ${ }^{22}$ describe a patient with a previously coiled anterior communicating artery and basilar artery apex aneurysm who presented with a recur- rent basilar apex aneurysm. She was pretreated with 10 days of aspirin/clopidogrel prior to stent assisted coiling of the aneurysm. During the procedure, in-stent thrombosis was observed in the right P1 and P2 segments that was treated with intravenous eptifibatide in addition to a $60 \mathrm{mg}$ oral load of prasugrel. The thrombus resolved on serial angiograms but the patient did have a retroperitoneal hematoma at the arteriotomy site requiring a blood transfusion. While it remains unclear which additional antiplatelet agent may have contributed to the hematoma, this report underscores both the necessity and potential risk of treatment with more potent thienopyridine agents.

While our series is the largest to date documenting the safety and efficacy of DAPT with aspirin/prasugrel in the neurointerventional setting, we acknowledge multiple limitations to our study. First, this study is a retrospective case series with a limited number of patients. Second, not all patients who were treated with either DAPT regimen received comparable doses of antiplatelet agents. It is therefore possible that subtle variations in dosing regimens between treatment groups could have impacted the overall rate of hemorrhage. Third, all procedures were performed by a single senior neurointerventionalist at a high volume academic institution with a low complication rate for neurointerventional procedures. As such, our results cannot necessarily be extrapolated to all interventionalists at any endovascular center. Fourth, our study is technically limited by a gold standard assay for platelet inhibition. While light transmission aggregometry is considered by some authorities to represent the current standard assay, ${ }^{23}$ this technique is expensive, labor intensive, requires specialized equipment and personnel, and is not readily available at many centers. Although newer point of care methodologies have shown clinical promise with regard to quantification of platelet inhibition, agreement between these assays to identify patients with sufficient platelet inhibition is low. ${ }^{24}$ While the senior author has adopted the VerifyNow assay based on its superior ability to quantify the biological activity of clopidogrel over other assays, ${ }^{25}$ we acknowledge that the lack of clear criteria for establishing platelet inhibition and the wide range of available tests are a limitation to our study. Finally, it deserves further mention that those patients who were treated with aspirin/prasugrel DAPT were discovered pre-procedurally to be clopidogrel nonresponders. As such, this represents a source of selection bias for our study.

In conclusion, our results suggest that in clopidogrel nonresponders, DAPT with aspirin/prasugrel may increase the risk of hemorrhage during neurointerventional surgery compared with DAPT with aspirin/clopidogrel. We recommend caution and meticulous microcatheter techniques when using antiplatelet regimens involving this agent. Clearly, further randomized investigations will be required to determine the clinical effects of 
clopidogrel resistance in the neurointerventional patient population and to validate the need for platelet inhibition laboratory testing in this subgroup. We look forward with enthusiasm to further research efforts in this area and to increased communication among neurointerventional surgeons about their experiences with these antiplatelet agents.

Contributors Conception and design: MRR and CJM; analysis and interpretation of the data: MRR, SHA and CJM; drafting the article: MRR, SHA and CJM; critically revising the article: all authors; reviewed final version of the manuscript and approved it for submission: all authors; administrative/technical/material support: MRR, SHA and CJM.

\section{Competing interests None}

Ethics approval Ethics approval was provided by Washington University Medical School Human Research Protection Office.

Provenance and peer review Not commissioned; externally peer reviewed.

Data sharing statement A synopsis of our original dataset is presented in the current paper. However, additional data, including explanatory material, complete data sets, etc, is available to fellow researchers on request.

Open Access This is an Open Access article distributed in accordance with the Creative Commons Attribution Non Commercial (CC BY-NC 3.0) license, which permits others to distribute, remix, adapt, build upon this work non-commercially, and license their derivative works on different terms, provided the original work is properly cited and the use is non-commercial. See: http://creativecommons.org/ licenses/by-nc/3.0/

\section{REFERENCES}

1. Qureshi Al, Luft AR, Sharma M, et al. Prevention and treatment of thromboembolic and ischemic complications associated with endovascular procedures: part II-clinical aspects and recommendations. Neurosurgery 2000;46:1360-75.

2. Kushner FG, Hand M, Smith SC Jr, et al. 2009 focused updates: ACC/AHA guidelines for the management of patients with ST-elevation myocardial infarction (updating the 2004 guideline and 2007 focused update) and ACC/AHA/SCAI guidelines on percutaneous coronary intervention (updating the 2005 guideline and 2007 focused update) a report of the American College of Cardiology Foundation/ American Heart Association Task Force on Practice Guidelines. J Am Coll Cardiol 2009:54:2205-41.

3. Mehta SR, Yusuf S, Peters RJG, et al. Effects of pretreatment with clopidogrel and aspirin followed by long-term therapy in patients undergoing percutaneous coronary intervention: the PCI-CURE study. Lancet 2001:358:527-33.

4. Smith SC Jr, Feldman TE, Hirshfeld JW Jr, et al. ACC/AHA/SCAI 2005 Guideline Update for Percutaneous Coronary Intervention-Summary Article: A Report of the American College of Cardiology/American Heart Association Task Force on Practice Guidelines (ACC/AHA/SCAI Writing Committee to Update the 2001 Guidelines for Percutaneous Coronary Intervention). J Am Coll Cardiol 2006:47:216-35.

5. Steinhubl SR, Berger PB, Mann JT 3rd, et al. Early and sustained dual oral antiplatelet therapy following percutaneous coronary intervention. JAMA 2002:288:2411-20.

6. Howington JU, Hanel RA, Harrigan MR, et al. The Neuroform stent, the first microcatheter-delivered stent for use in the intracranial circulation. Neurosurgery 2004;54:2-5.
7. Qureshi Al, Luft AR, Sharma M, et al. Prevention and treatment of thromboembolic and ischemic complications associated with endovascular procedures: part I-pathophysiological and pharmacological features. Neurosurgery 2000:46:1344-59.

8. Gurbel PA, Bliden KP, Hiatt BL, et al. Clopidogrel for coronary stenting. Circulation 2003:107:2908-13.

9. Serebruany VL, Steinhubl SR, Berger PB, et al. Variability in platelet responsiveness to clopidogrel among 544 individuals. J Am Coll Cardiol 2005;45:246-51.

10. Buonamici P, Marcucci R, Migliorini A, et al. Impact of platelet reactivity after clopidogrel administration on drug-eluting stent thrombosis. J Am Coll Cardiol 2007;49:2312-17.

11. Hochholzer W, Trenk D, Bestehorn HP, et al. Impact of the degree of periinterventional platelet inhibition after loading with clopidogrel on early clinical outcome of elective coronary stent placement. J Am Coll Cardiol 2006:48:1742-50.

12. Niitsu Y, Jakubowski JA, Sugidachi A, et al. Pharmacology of CS-747 (prasugrel, LY640315), a novel, potent antiplatelet agent with in vivo P2Y12 receptor antagonist activity. Semin Thromb Hemost 2005;31:184-94.

13. Dobesh PP. Pharmacokinetics and pharmacodynamics of prasugrel, a thienopyridine p2y12 inhibitor. Pharmacotherapy 2009;29:1089-102.

14. Mahoney EM, Wang K, Arnold SV, et al. Cost-effectiveness of prasugrel versus clopidogrel in patients with acute coronary syndromes and planned percutaneous coronary intervention results from the trial to assess improvement in therapeutic outcomes by optimizing platelet inhibition with Prasugrel-Thrombolysis in Myocardia Infarction TRITON-TIMI 38. Circulation 2010;121:71-9.

15. Farid NA, Smith RL, Gillespie TA, et al. The disposition of prasugrel, a novel thienopyridine, in humans. Drug Metab Dispos 2007;35:1096-104.

16. Weerakkody GJ, Jakubowski JA, Brandt JT, et al. Comparison of speed of onset of platelet inhibition after loading doses of clopidogrel versus prasugrel in healthy volunteers and correlation with responder status. Am J Cardiol 2007:100:331-6.

17. Wiviott SD, Braunwald $\mathrm{E}, \mathrm{McC}$ abe $\mathrm{CH}$, et al. Intensive oral antiplatelet therapy for reduction of ischaemic events including stent thrombosis in patients with acute coronary syndromes treated with percutaneous coronary intervention and stenting in the TRITON-TIMI 38 trial: a subanalysis of a randomised trial. Lancet 2008; 371:1353-63

18. Wiviott SD, Braunwald E, McCabe $\mathrm{CH}$, et al. Prasugrel versus clopidogrel in patients with acute coronary syndromes. N Engl J Med 2007;357:2001-15.

19. Armero S, Bonello L, Berbis J, et al. Rate of nuisance bleedings and impact on compliance to prasugrel in acute coronary syndromes. Am J Cardiol 2011;108:1710-13

20. Wiviott SD, Antman EM, Gibson CM, et al. Evaluation of prasugrel compared with clopidogrel in patients with acute coronary syndromes: design and rationale for the TRial to assess Improvement in Therapeutic Outcomes by optimizing platelet InhibitioN with prasugrel Thrombolysis In Myocardial Infarction 38 (TRITON-TIMI 38). Am Heart J 2006;152:627-35.

21. Kalyanasundaram A, Lincoff AM. Managing adverse effects and drug-drug interactions of antiplatelet agents. Nat Rev Cardiol 2011;8:592-600.

22. Leslie-Mazwi TM, Chandra RV, Oh DC, et al. Novel use of prasugrel for intracranial stent thrombosis. J Neurointerv Surg. Published Online First: 18 February 2011. doi 10.1136/jnis.2010.004382

23. Michelson AD. Platelet function testing in cardiovascular diseases. Circulation 2004:110:e489-93.

24. Lordkipanidzé M, Pharand C, Nguyen TA, et al. Comparison of four tests to assess inhibition of platelet function by clopidogrel in stable coronary artery disease patients. Eur Heart J 2008;29:2877-85.

25. Bouman H, Parlak E, Van Werkum J, et al. Which platelet function test is suitable to monitor clopidogrel responsiveness? A pharmacokr!ejiz a.าalysis or! th.e astive metabolite of clopidogrel. J Thromb Haemostasis 2010;8:482-8. 